\section{A digital programmer for use with audio tape decks*}

\author{
RONALD H. HOPKINS† \\ Washington State University, Pullman, Washington 99163
}

A flexible inexpensive programming device for use with a magnetic tape deck is described. The programmer provides independent control for up to 15 different experimental events. It can be constructed from digital ICs for less than $\$ 50$ and may be used with an inexpensive tape deck. A general-purpose transistor switch is also described.

Studies of human learning and memory often require presentation of both auditory and visual stimuli. A common procedure is to present auditory stimuli via tape recorder and visual stimuli by filmstrip or slide projector. Any necessary synchronization is then generally obtained by detection of prerecorded tones to operate the projectors. If more than one event is to be programmed, several different tone generators and filters are required; this method, then, becomes somewhat complicated and very expensive. The encoder-decoder combination described here permits precise and reliable programming of up to 15 different events. ${ }^{1}$ This programmer takes advantage of the low cost of digital integrated circuits (ICs) and can be used with an inexpensive tape deck. The unit can be built for less than $\$ 50$.

\section{PRINCIPLES OF OPERATION}

During encoding, a train of pulses is recorded on one channel of the tape; during decoding, the number of pulses in a train is counted and the corresponding event is signaled. More specifically, a 4-bit binary number, representing an event code, is stored in an up/down counter of the encoder; then the counter is stepped to zero and one pulse is recorded with each step. During decoding, the first pulse of a train fires a one-shot whose output pulse width slightly exceeds the duration of the longest possible pulse train; all pulses entering the decoder during the period of this one-shot are treated as members of the same pulse train. This scheme permits discriminating the pulses of one train from the pulses of immediately

*This research was supported by United States Public Health Service Grant MH 20232-01, by Basic Research Grant A ward OEG-X-71-0032(508) from the United States Office of Education, and by graduate school research funds.

tRequests for reprints should be sent to Ronald H. Hopkins, Department of Psychology, Washington State University, Pullman, Washington 99163. preceding or following trains. The pulses of a given train are counted into a binary counter and the contents of the counter are decoded at the end of the train, signaling one of the 15 events.

Obviously, two events cannot be programmed to occur simultaneously by these operations. However, two events can readily be programmed with an interevent interval as short as $20 \mathrm{msec}$, sufficient for much of the programming in psychological research. The limiting factor determining the minimum interevent interval is the frequency of the pulse generator, which is, in turn, limited by the transient frequency response of the tape deck. For an inexpensive deck of the type used in our laboratory (Sony TC-366), good reliability is provided by using a $1-\mathrm{KHz}$ pulse generator. With a higher quality tape deck, it might be possible to use a frequency as high as 10 or $12 \mathrm{KHz}$, thereby reducing the minimum interevent interval by a factor of 10 . Even a high-quality deck, however, is likely to have poor square-wave response above $5 \mathrm{KHz}$. This makes it difficult to discriminate the code pulses from noise spikes on the magnetic tape and results in reduced reliability.
ENCODER

The logic of encoder operation is outlined in the left panel of Fig. 1. Each functional unit will be referred to by the number indicated in Fig. 1 for that unit (1 to 26).

The heart of the encoder is the binary up/down counter (9). The inputs to the counter are made via one-shots (5-8) which are, in turn, fired by four push buttons for manual programming or by four remote switch closures to ground. The potential effects of contact bounce in the push buttons are eliminated by connecting each push button as a bounceless switch using flip-flops (1-4). The input one-shots are set for a pulse width of about $4 \mathrm{msec}$ to reduce the effects of contact bounce in remote inputs. If the bounce in remote inputs exceeds 4 msec, the duration of the one-shot inputs should be lengthened by varying the external resistance and capacitance on each one-shot. Notice that the input can be any 4-bit binary number, permitting the encoding of up to 15 different events (excluding zero).

As soon as any number, other than zero, is detected in the counter by the detect gates (10-14), a delay one-shot (15) of about $3 \mathrm{msec}$ duration is fired. This delay permits nominally simultaneous inputs which are asynchronous by as much as $3 \mathrm{msec}$. The duration of this delay must be less than that of the input one-shots $(5-8)$ (when the input one-shots time out, the data are no longer present and only zeroes will be stored in the counter). It is difficult to depress manually two or more push buttons within $3 \mathrm{msec}$ of one another; consequently, if you anticipate that many codes will require simultaneous entries from more than one push button, the pulse widths of the input one-shots (5-8) and of One-Shot 15 should be lengthened appropriately. After it times out, One-Shot 15 fires

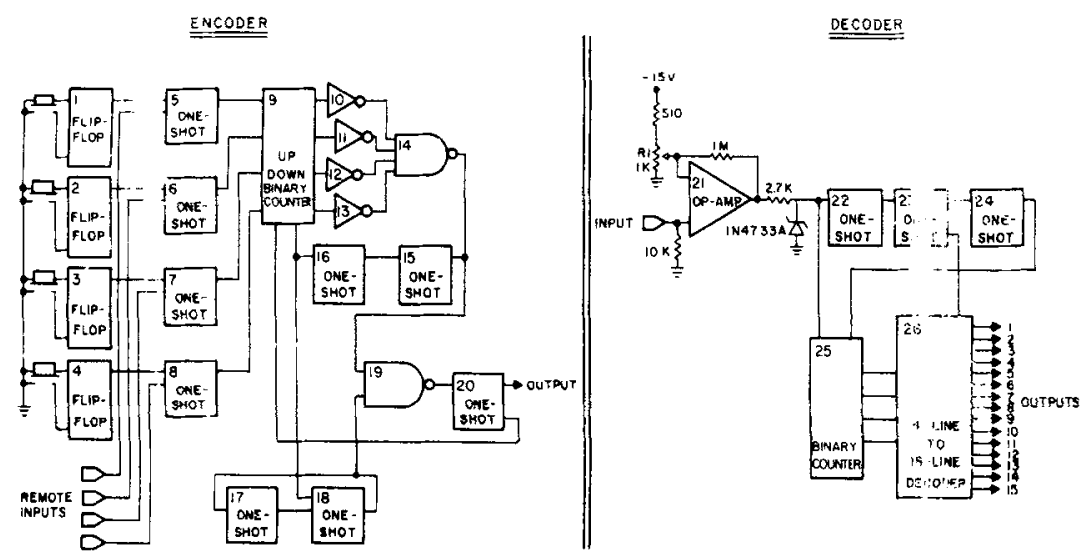

Fig. 1. Logical functions of the encoder and decoder. 


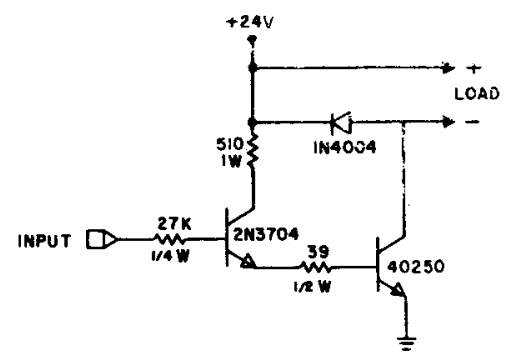

Fig. 2. A general-purpose transistor switch.

an enabling one-shot (16), which enables the pulse generator $(17-18)$ and disenables the load function of the counter; this prohibits the entry of a new code. The pulse width of One-Shot 16 should be slightly greater than the duration of the longest pulse train; in this case, it was set for $19 \mathrm{msec}$. As long as the content of the counter (9) is not equal to zero and the enabling one-shot (16) has not timed out, each pulse from the pulse generator (17-18) is gated (19) to the output one-shot (20), which outputs one pulse and decrements the counter (9) by one. This configuration precludes the possibility that detection of zero in the counter will prematurely terminate the last pulse of a train. The high input impedance of a tape deck permits direct connection of the output from One-Shot 20 to the input of the tape deck.

(In the present application, the pulse generator was set to produce a 0.04 -msec pulse once each millisecond by setting the pulse width of One-Shot 17 at $1.0 \mathrm{msec}$ and the pulse width of One-Shot 18 at $0.04 \mathrm{msec}$. The pulse width of One-Shot 20 was adjusted to $0.1 \mathrm{msec}$, so the output of the encoder is a $0.1-\mathrm{msec}$ step to ground potential each $1.0 \mathrm{msec}$ for the duration of the pulse train. These parameters have worked well with the detection circuit used in the decoder.)

\section{DECODER}

The logical operation of the decoder is diagrammed in the right panel of Fig. 1. The first section of the decoder is a level detector, made from an op-amp (21), which discriminates the pulses from background noise spikes. The sensitivity of the level detector is adjusted by $R 1$, and the output is clamped at $+5 \mathrm{~V}$ by the Zener diode. The first pulse of a train enters the binary counter (25) and also fires One-Shot 22. The pulse width of One-Shot 22 is adjusted to be slightly greater than the duration of a train of 15 pulses, i.e., about $20 \mathrm{msec}$. All succeeding pulses enter the counter until One-Shot 22 times out, firing One-Shot 23. The latter one-shot enables the output of the 4-to-16-line decoder (26) momentarily, and then fires One-Shot 24, which provides a $0.001-\mathrm{msec}$ pulse to reset the counter in preparation for the next pulse train. The 4-to-16-line decoder (26) is simply a series of gates which converts the binary output of the counter (25) to a decimal output from 1 to 15 .

All outputs from the decoder are at $+5 \mathrm{~V}$ until One-Shot 23 fires; then the one line of the decoder corresponding to the contents of the counter drops to ground potential for the duration of the pulse from One-Shot 23. In the present application, the output of the decoder is simply used to operate other IC components, and One-Shot 23 has a pulse width of 0.13 msec. Obviously, if the output is to operate a relay, the pulse must be longer and a suitable driver is required (see description of transistor switch below). Lengthening of the output pulse does, of course, increase the minimum interval between pulse trains. In any case, the output of the decoder ordinarily must be only of sufficient duration to activate external timers. Obviously, if one uses One-Shot 23 to time external events as well as signal their occurrence, those events will all have the same duration.

\section{TRANSISTOR SWITCH}

It is often necessary to switch heavy loads, such as relays or solenoids, from IC components. An engineer can diagram a basic transistor switch, but it is often difficult to determine specific component values for a given purpose. Since it is likely that many users of this programmer would need such switches, a specific example is provided in Fig. 2. This transistor switch has worked well with the $24-\mathrm{V}$ loads used in psychology laboratories. The switch uses inexpensive transistors and can switch a .75-A 24-V load with only a $0.2-\mathrm{mA} 3-\mathrm{V}$ input (well within the fanout capabilities of TTL logic). The current capability of the 40250 is about $4 \mathrm{~A}$, and loads heavier than $0.75 \mathrm{~A}$ can be handled by cascading another $2 \mathrm{~N} 3704$ at the input or by decreasing the current-limiting $27 \mathrm{~K}$ resistor, thereby permitting larger current input to the driver. Most TTL outputs can handle at least $10 \mathrm{~mA}$. Note that the load operates when the input to the switch is positive, so the outputs of the decoder would have to be inverted with an $5 N 7404 \mathrm{~N}$ or similar inverter. Loads between 10 and $500 \mathrm{~mA}$, such as many incandescent lamps and relays, can be operated by a buffer driver such as the $\$ \mathrm{~N} 7407 \mathrm{~N}$ or the recently available IC-compatible reed relays. The latter have the advantage of providing complete isolation between the IC circuitry and the load.

\section{CONSTRUCTION}

The point-to-point wiring diagrams

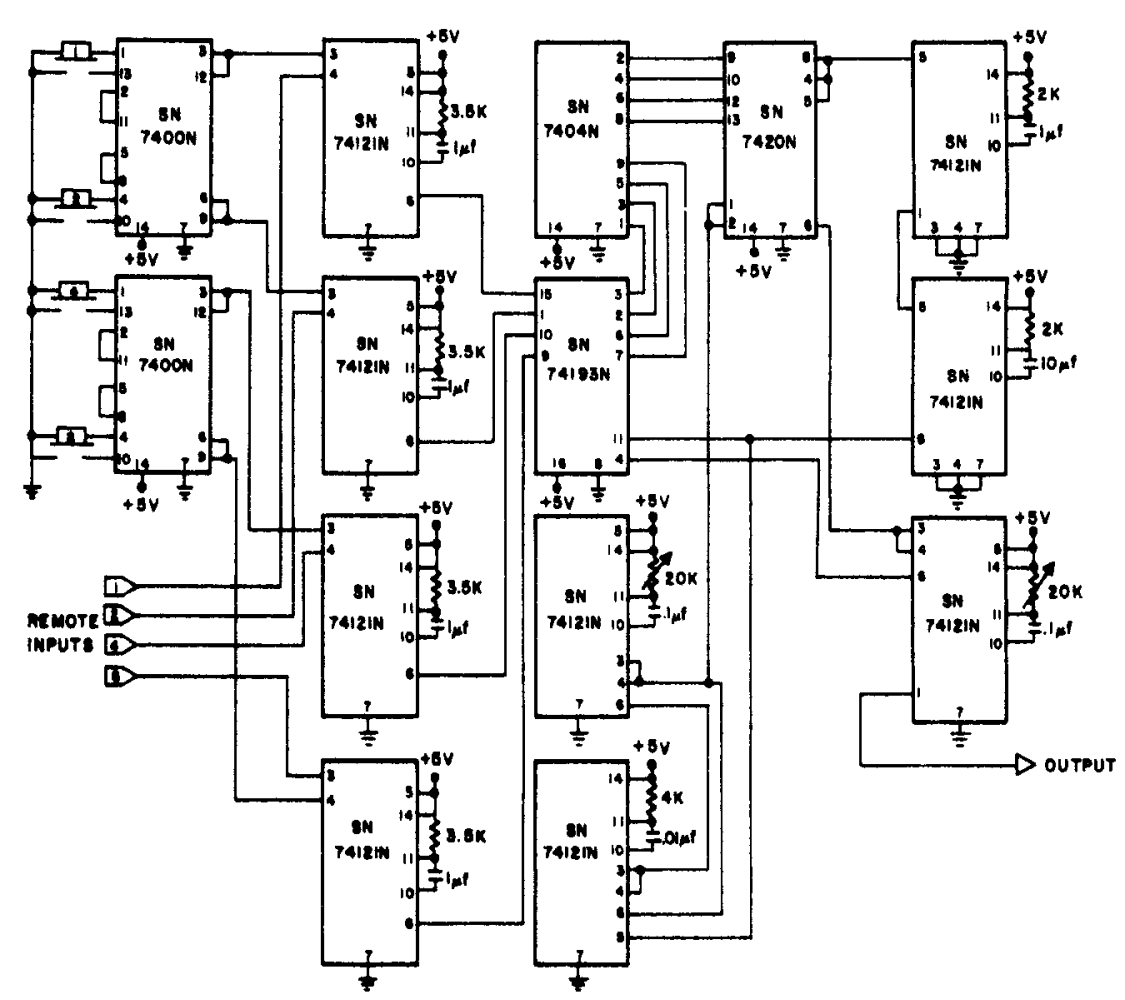

Fig. 3. Point-to-point wiring of the encoder. 


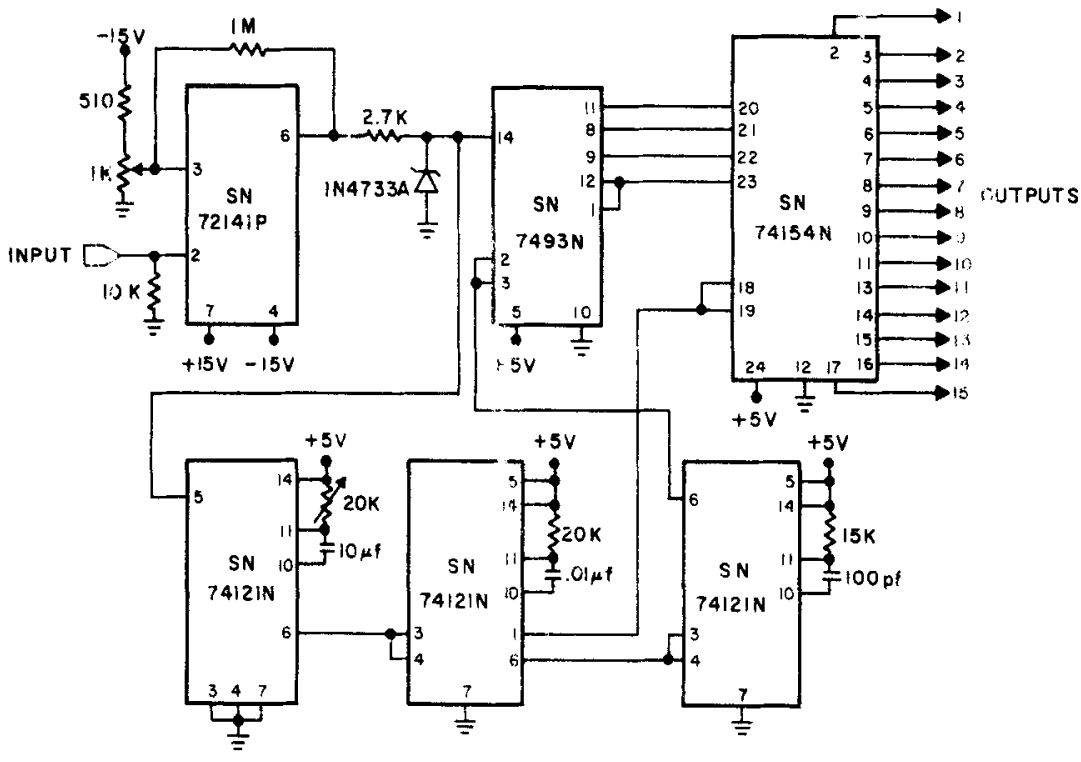

Fig. 4. Point-to-point wiring of the decoder.

are shown in Fig. 3 (encoder) and Fig. 4 (decoder). The layout doesn't seem to be critical. The prototype was constructed on 0.1-in. center perforated board, with push-in terminals used as interconnection points. All ICs were socketed. The $5-\mathrm{V}$ power was bussed along one edge with .01 and $10-\mathrm{microF}$ decoupling capacitors about every five IC packages. All variable resistors were 20-turn trim pots. The encoder and decoder were assembled on separate perforated boards and attached perpendicular to rack panels on which connectors and controls were mounted. Ordinary shielded audio cable and connectors were used between the encoder and tape recorder, and between the tape recorder and decoder. The other connectors were all miniature banana jacks, but any type of connector will do. The reader is referred to Morris and Miller (1971) and to manufacturer's catalogs (e.g., Texas Instruments, 1971) for further information and specifications of the ICs used.

\section{CALIBRATION AND ADJUSTMENT}

A scope with calibrated time base was used for testing and adjustment of all one-shots. The recording and playback levels of the tape recorder, as well as the sensitivity of the level detector, were also adjusted. For this purpose, it was convenient to first connect the scope to the tape-recorder output, play the recorder in the record mode, and manually enter various codes while adjusting the record and playback levels for maximal fidelity of the recorder output to the pulse-train input (a dual trace scope is useful, but certainly not necessary, for this operation). The recorder should be operated at $19 \mathrm{~cm} / \mathrm{sec}$. The optimal settings are then used for all recordings. The scope was then connected to the output of the level detector, and R1 was adjusted until each pulse was detected, with no noise spikes falsely detected. If the recording and playback levels are appropriately adjusted, there should be substantial margin for error in the setting of $\mathrm{R} 1$, particularly with a pulse generator frequency as low as $1 \mathrm{KHz}$.

\section{RECORDING}

One drawback of a magnetic tape programmer of any type is that the recordings must be made in real time. With paper tape, it is a simple matter to space the code holes for control of interevent intervals; with magnetic tape, the tape must be in motion during the recording process and the interevent intervals must be dynamically recorded. Pushing the correct encoding buttons at the appropriate time is rather difficult if accuracy is important and/or if the program is complex; however, this method has been used successfully with simple programs. If the recorder has a reasonably instantaneous start, and if the precision required is not too great, it is possible to control interevent intervals by stopping the recorder after each code, advancing the appropriate distance along the tape, starting the recorder just prior to entering the next code, etc. It is convenient to use an external program source (such as a stepping switch or paper tape reader stepped at the appropriate rate) to put the event codes in permanent form on the tape via the remote inputs. Regardless of the method for recording the event codes on tape, the pulse trains conveniently leave an audible blip on the program channel. Monitoring these blips with headphones while recording stimulus words on the other channel enables the user to obtain adequate synchronization between event codes and auditory stimulus words. If the auditory stimuli are simple tones, they might be recorded at the same time as the programming information.

\section{COST}

The ICs for the encoder and decoder list at about $\$ 40$, but can be purchased from numerous sources for about $\$ 15$. The entire system, including hardware and discrete components, can be assembled for less than $\$ 45$ if power supplies are available. Many labs have $5-$ and $\pm 15-\mathrm{V}$ power supplies; if not, they can be purchased for approximately $\$ 20$ each. Current capability of the power supplies needs not be high, but it should be regulated.

\section{APPLICATIONS}

The programmer can be used in any application requiring a flexible, inexpensive, stored-program device, whether auditory stimuli are required or not. It is, of course, most likely to be used in situations requiring the use of auditory stimuli in conjunction with other types of stimulus events. The prototype system has been in operation for 1 year, and about 1,000 Ss have been run in verbal short-term memory experiments requiring two slide projectors, with external shutters, to be synchronized with auditory stimuli. The program for the slide projectors is recorded on one channel and the auditory stimuli on the other channel of a stereo tape deck (Sony TC-366). In a typical experiment, four of the possible 15 event codes are used. Code 1 sets a control flip-flop which operates a driver and opens Shutter 1 . Code 2 resets Flip-Flop 1, closing Shutter 1, and also fires a one-shot which operates a driver-relay combination to advance Projector 1 , readying that projector for its next presentation. Code 3 sets Control Flip-Flop 2, opening Shutter 2; Code 4 resets Flip-Flop 2, closing Shutter 2 and advancing Projector 2. Reliability has been extremely high.

\section{REFERENCES}

MORRIS, R, L \& MILLER, J. R. Designin with TTL integrated circuits. New York: McGraw-Hill, 1971.

TEXAS INSTRUMENTS. The integrated circuits catalog for design engineers. Dallas: Texas Instruments, Inc., 1971.

\section{NOTE}

1. There are other schemes for recording digital information on audiotape, e.g. Lentz, R., \& Haith, M, M. Audio tape storage of experimental data: An application to tachistoscopic research with children. Behavior Research Methods \& Instrumentation, 1969, 1, 273-275. 